\title{
Género y formación del profesorado. Propuestas del profesorado en formación para introducir a las mujeres en el currículo de Ciencias Sociales ${ }^{1}$
}

\author{
Helena RAUSELL GUILLOT \\ Rafael VALLS MONTES
}

Datos de contacto:

Helena Rausell Guillot Universitat de València Helena.Rausell@uv.es

Rafael Valls Montes Universitat de València Rafael.Valls@uv.es

Recibido: 16/07/2020 Aceptado:12/01/2021

\begin{abstract}
RESUMEN
Este artículo investiga las formas que adopta la inclusión de la perspectiva de género en las propuestas de los estudiantes del Máster de Profesor/Profesora de Educación Secundaria de la Universidad de Valencia. La investigación forma parte de un estudio más amplio sobre las representaciones sociales y las prácticas del futuro profesorado de secundaria en formación. Se enmarca dentro de la metodología cualitativa y el estudio colectivo de casos. El instrumento de análisis son una serie de cuestionarios que fueron implementados en dos cursos sucesivos (2016-2017 y 2017 2018) para una muestra de 69 alumnos. Las respuestas han sido analizadas en función de variables como el ámbito cronológico y disciplinar consignado o su adecuación a las propuestas que proporciona la bibliografía especializada sobre las posibilidades de incorporación de las mujeres al currículo. Los resultados muestran la relevancia de la formación previa en género, el peso de la formación disciplinar inicial, así como la falta de especificidad de muchos de los diseños, que, sin embargo, tienden a reproducir, simplificadas, las aportaciones del género y de la historia de las mujeres.
\end{abstract}

PALABRAS CLAVE: Género; Formación del Profesorado; Currículo; Ciencias Sociales

\footnotetext{
1 Este trabajo forma parte del proyecto de investigación PGC2018-094491-B-C32, financiado por el Ministerio de Ciencia, Innovación y Universidades y cofinanciado con fondos FEDER de la UE.
} 


\title{
Gender and teacher training. Proposals of trainee teachers to introduce women into the Social Sciences curriculum
}

\begin{abstract}
This article investigates how the students of the Master's Degree in Secondary Education Teaching (University of Valencia, majoring in Geography and History) design the inclusion of the gender perspective in Social Sciences. The research is part of a larger study on the social representations and practices of trainee teachers. It is framed within the qualitative methodology and the collective cases study. Discourse and representations related to gender and curriculum will be analyzed and categorized by means of initial surveys. These surveys were implemented in successive academic years (2016-2017 and 2017-2018) for a sample of 69 students. The analysis is based on variables such as the chronological and disciplinary field. It also takes into account possibilities provided by literature in order to incorporate women into the curriculum. The results show the relevance of previous gender and initial disciplinary training, as well as the lack of specificity of many of the designs, which, however, tend to reproduce, in a simplified way, the contributions of gender and women's history and art history.
\end{abstract}

KEYWORDS: Gender; Teacher Training; Curriculum; Social Sciences

\section{Introducción}

Los cambios introducidos en las últimas décadas en los sistemas educativos occidentales han ido eliminando los aspectos discriminatorios que eran más explícitos o evidentes en relación a las mujeres. Pese a ello, se mantienen otros muchos como el sexismo, la invisibilidad de las mujeres o la distribución estereotipada de jóvenes estudiantes y de docentes en diferentes ramas de estudios dentro del sistema educativo (Anguita \& Torrego, 2009). En su base, las innegables carencias que, en materia de género, evidencian las últimas reformas educativas:

1. La falta de concreción de las leyes educativas, que proclaman la igualdad, pero que, amparándose en su transversalidad, no la concretan, de tal modo que no hay materiales ni asignaturas específicas, tampoco créditos asignados, ni consignas para que se incluya en evaluación ni en las prácticas (Anguita \& Torrego, 2009; Anguita, 2001; Donoso \& Velázquez, 2013).

2. El convencimiento de que la igualdad real entre hombres y mujeres es ya un hecho.

3. Las gravísimas carencias formativas en materia de género del profesorado en formación (Rebollar, 2013; Ibeas \& Garrido, 2018). 
El feminismo académico "ha producido uno de los cambios más transformadores, creativos e importantes dentro de la práctica y la política académica, lo que no implica que, a nivel docente, exista una oferta acorde con la cantidad de información y conocimiento desarrollado" (Donoso \& Velázquez, 2013: 74). El sistema universitario sigue privilegiando a los hombres y eso a pesar de que la población femenina con estudios universitarios es superior a la masculina desde $2006 \mathrm{y}$ a pesar también del hecho de que, a fecha de marzo de 2019, el 66,5\% de todo el profesorado de nuestro país es femenino, un porcentaje que aumenta al 71,9\% en las enseñanzas no universitarias y se desploma hasta el $41,3 \%$ en la educación universitaria. ${ }^{2}$

\section{Marco teórico}

La formación del profesorado desde una perspectiva de género sigue siendo una asignatura pendiente, tanto en lo referente a la formación inicial como en la formación permanente. Diferentes estudios denuncian su escasa presencia en algunas de las guías docentes de los grados y postgrados de educación (Jaramillo, 1999; Rebollar, 2013; Rausell \& Talavera, 2017). Las Ciencias Sociales - Historia, Geografía e Historia del Arte - han sido, por su parte, especialmente lentas a la hora de incorporar el género a su docencia (Crocco, 1997), sobre todo fuera del ámbito estrictamente universitario, al haber mantenido una aproximación sexista y patriarcal acerca de qué conocimiento es considerado importante (Minnich,1990; Crocco, 2004).

En el campo de la Historia del Arte y de su didáctica, son fundamentales los trabajos de Nochlin $(1971,1989)$, Parker \& Pollock (1981), Nead (1998), López F.Cao (2001) o Hustvedt (2017), entre otros. Como en otras disciplinas, el primer avance se centró en la recuperación de las mujeres creadoras, aunque la revisión feminista "suponía cuestionar muchas de las categorías, como las de «genio artístico», «calidad» o «influencia», sobre las que asentaba la disciplina de la Historia del Arte en su conjunto" (Mayayo, 2003: 13). Igualmente, denunciaba la forma en la que las artes visuales están estructuradas en torno al espectador masculino, describiendo el mundo y las mujeres desde el punto de vista del varón (Mulvey, 1989; Berger 1972). Dentro de la Geografía, se ha analizado la forma en la que el discurso geográfico ha sido importante en la decodificación feminista de las visiones del mundo. También la forma sobre cómo las relaciones patriarcales generan experiencias género-específicas de tiempo y espacio (Del Valle, 1991; Bondi, 1993), imbricándose con diferentes movimientos políticos que denuncian las desigualdades de género a escala mundial y que se conectan, además, con las identidades LGBTQ+ (Wright, 2008 y 2009). Por su parte, la inclusión de la asignatura de Historia de las

\footnotetext{
2 Ministerio de Educación y Formación Profesional (2019): Igualdad en cifras. http:/ / www.educacionyfp.gob.es/dam/jcr:957c29bb-ebd1-4e5b-9417$\underline{3 d 163 c c 32 d e f / c i f r a s w e b . p d f}$
} 
mujeres en los grados de algunas facultades de Historia y la creación de postgradoos específicos vinculados a los Institutos Universitarios de Estudios e Investigación de las Mujeres y del Género (Bolufer, 2018) han sido muy importantes para la investigación y la educación universitarias en materia de género. Sin embargo, dichos estudios no están presentes en todas las universidades españolas ni tienen su equivalente en los grados de educación.

Una de las grandes cuestiones pendientes es la forma de incorporar a las mujeres al currículo de la Historia, la Historia del Arte o de la Geografía. En este sentido y en relación a la Historia, destacan los trabajos de Lerner (1979, 1993), McIntosh (1983) y Woyshner (2002), de los que se hacen eco, entre otros, Pages y Sant (2012). Estos estudios proporcionan el marco de análisis de la investigación que, como señalábamos supra, estudia la forma que adopta la inclusión de la perspectiva de género en las propuestas del alumnado del Máster de Profesor/Profesora de Educación Secundaria de la Universidad de Valencia (en adelante MAES).

\section{Método}

La investigación forma parte de un estudio más amplio sobre las representaciones sociales y prácticas del futuro profesorado de secundaria en formación, centrado en el caso concreto de los alumnos del Master de Formación de Profesor/Profesora de Enseñanza Secundaria de la Universidad de Valencia. Se enmarca dentro de la metodología cualitativa y en relación con el paradigma crítico y el feminismo. Se trata de un estudio colectivo de casos (Stake, 2007; Simons, 2011) que interroga acerca de las posibilidades de incluir a las mujeres en la enseñanza de la Historia, la Geografía y la Historia del Arte, las finalidades de las Ciencias Sociales y la ausencia/presencia del género en la formación inicial y didáctica del profesorado de secundaria o sobre los propios fines de la educación.

\section{La metodología cualitativa y el diseño de los instrumentos de recogida de resultados}

El paradigma cualitativo posibilita la aproximación a los problemas vinculados a la acción y la crítica social, pero también a aquellas cuestiones relacionadas con la didáctica de los invisibles, proporcionando una visión crítica y comprometida de la investigación (Taylor \& Bodgan, 1984). El instrumento de análisis empleado es una serie de cuestionarios (pre-test, intermedio y final o de auto-evaluación) en dos cursos sucesivos (2016-2017 y 2017-2018) para una muestra de 69 estudiantes.

Los cuestionarios respetan el anonimato de los y las participantes e incluyen diferentes variables de control. La encuesta se entiende como un instrumento de recogida de datos, pero también como un método de investigación que abarca la formulación del problema, el establecimiento de los objetivos, la selección de los sujetos y el diseño y análisis de datos (Alaminos \& Castejón, 2006). Por lo que respecta a su dimensión temática (Álvarez-Gayou, 2003; Cohen \& al., 2007), la 
conceptualización del cuestionario inicial está relacionada con la formación en género del futuro profesorado de secundaria. Se articula en una triple dimensión: formación inicial, dimensión valorativa-actitudinal y diseño de las intervenciones e incluye hasta cinco indicadores diferentes, que se corresponden a los siguientes ítems: formación recibida en género; conocimientos previos; valoración personal; predisposición para incorporar el género a su futura docencia $y$, finalmente, posibilidades contempladas para la introducción de las mujeres en el curriculum. Los objetivos que perseguíamos con el diseño e implementación de este cuestionario inicial eran los siguientes:

- Saber si los futuros docentes de enseñanza secundaria habían recibido o no formación específica sobre género.

- Ver cuáles eran las definiciones aportadas para el concepto de género. Identificar qué elementos incorporaban a sus definiciones (el género como construcción social y cultural, su carácter perfomativo, la conexión con la teoría queer...).

- Conocer su valoración de las aportaciones de la perspectiva de género a la Geografía, la Historia, la Historia del Arte y sus didácticas. Saber si colocaban dichas aportaciones en un nivel de relevancia similar a otras.

- Conocer su postura frente a la posibilidad de introducir el género en el ejercicio de la profesión docente. Analizar cómo expresan por escrito la necesidad de incorporar el género a la enseñanza de las ciencias sociales y en qué términos la justifican.

- Conocer sus propuestas para hacerlo y a qué períodos o a qué ámbitos temáticos de la Historia, la Historia del Arte o la Geografía remiten.

\section{El contexto de la investigación y los datos de la muestra}

El contexto de la investigación es un título de postgrado de 60 créditos con una orientación profesionalizadora que habilita para el ejercicio de las profesiones reguladas de profesor de Enseñanzas Obligatorias y Bachillerato, Formación Profesional y Enseñanza de Idiomas. En su diseño se trataba de mejorar la capacitación inicial de los docentes que, hasta ese momento, respondía a una lógica básicamente disciplinar y bastante desligada de las funciones educativas (Hernández \& Carrasco, 2012). La opción rechazaba el modelo concurrente, perpetuando la dualidad entre contenido disciplinar y formación psico-pedagógica (Manso \& Martín, 2014; González-Sanmamed, 2015).

La muestra está constituida por 69 alumnos del MAES que cursaron la asignatura de «Complementos para la formación disciplinar» en 2016-2017 y 2017-2018 dentro del grupo de mañanas de la especialidad de Geografía e Historia. Se trata, en su mayoría, de alumnado que han finalizado el grado de Historia (59'98\%), Historia del Arte (21'42\%) y Geografía y Medio Ambiente (10\%), con un porcentaje residual de estudiantado procedentes de otros grados (Humanidades, Sociología o Periodismo). Respecto a la variable género, son más hombres que mujeres (54'26\% frente a $\left.45^{\prime} 69 \%\right)$, con una edad media algo inferior a los 24 años. Aunque en el cuestionario 
incluimos la opción "otros" a fin de no presentar solo dos versiones del género ligadas al binario masculino - femenino, ninguno de los participantes marcó dicha casilla al cumplimentar los datos iniciales. Los resultados muestran que más de la mitad de los encuestados (56’5\%) han recibido formación específica en género, esto es, han cursado al menos una asignatura consagrada exclusivamente a ella. Este porcentaje se eleva hasta el 76'85\% de la muestra si consideramos a aquellos que han abordado cuestiones de género de forma transversal. Aquellos que no han tenido ninguna formación de género son el 23’2\%.

\section{Preguntas de investigación y objetivos}

La pregunta que analizamos forma parte del cuestionario inicial, que está integrado por cinco preguntas abiertas ${ }^{3}$. En este artículo presentaremos los resultados de las preguntas cuatro y cinco, que interrogan directamente a los alumnos sobre la intención de incorporar el género a su futura docencia, además de pedirles que diseñen, en unas pocas líneas, una propuesta de intervención en el aula justificando su elección ${ }^{4}$. Se sitúa con ello, en la intersección de dos de las tres dimensiones temáticas del cuestionario inicial indicadas supra: la dimensión valorativa-actitudinal y el diseño de las intervenciones. Pondera la predisposición de los participantes a incorporar el género en su docencia y analiza las posibilidades que contemplan para introducir a las mujeres en el currículo. Dichas preguntas están, a su vez, relacionadas con los objetivos y las finalidades de la investigación, en especial la voluntad de contrastar y reflexionar en torno al discurso que expresan sobre la inclusión de las mujeres al currículo de Ciencias Sociales - Historia, Geografía, Historia del Arte - los docentes en formación.

\section{Las categorías de análisis}

Las categorías de análisis que utilizamos surgen al adaptar para nuestra investigación las propuestas de incorporación de las mujeres al currículo que la historiografía especializada plantea para la Historia (Lerner, 1979 y 1993; McIntosh, 1983, Woyshner, 2002; Pages y Sant, 2012). Se trataría, de este modo, de las siguientes:

1. La Historia, la Geografía y la Historia del Arte sin mujeres, una concepción disciplinar pretendidamente universal y sin embargo concebida desde una perspectiva androcéntrica, occidental y heterosexual sin presencia de mujeres. Se correspondería con lo que Lerner (1979) denomina male-

\footnotetext{
${ }^{3}$ El enunciado de las tres primeras preguntas es el siguiente: 1) ¿Conoces el concepto de género? ¿Podrías definirlo?; 2) ¿Has cursado alguna asignatura de género en tus estudios? ¿Cuál?; 3) ¿Consideras interesante la perspectiva de género para las Ciencias Sociales (historia, geografía, historia del arte)? ¿Por qué?;

${ }^{4}$ Dichas preguntas están enunciadas en los siguientes términos: 4) ¿Incorporarías a tu trabajo como futuro profesor de secundaria la perspectiva de género?; 5) ¿Cómo lo harías? Diseña en unas pocas líneas una propuesta o, al menos, enúnciala y justifica tu elección.
} 
defined history o McIntosh (1983) womanless, all-wite history.

2. La Historia, la Geografía y la Historia del Arte con las mujeres. En este enfoque se produce una adición a unas disciplinas aún androcéntricas, de modo que se subrayan las contribuciones de las mujeres a una sociedad aún definida en términos masculinos (Lerner, 1979), al tiempo que se les atribuye el rol de mujer masculinizada (Pagès \& Sant, 2012).

3. La Historia, la Geografía y la Historia del Arte de las mujeres. Se correspondería con el women on their own terms history del que hablara Lerner (1993), además de incorporar ámbitos y temáticas consideradas "femeninas" o de poner el énfasis en hechos y cuestiones relevantes para el feminismo.

4. La Historia, la Geografía y la Historia del Arte desde la perspectiva de las mujeres (Lerner, 1993; Woyshner, 2002). Son propuestas que explican las sociedades presentes y pasadas en clave de género, analizando la presencia de las mujeres en todos los ámbitos a partir de sus formas de participación, de exclusión o de integración, su presencia en la esfera pública, su participación en el poder político... Igualmente, la evolución de las construcciones de femenino y masculino en el tiempo, junto con el cuestionamiento de los fundamentos conceptuales, cronológicos y epistemológicos de las disciplinas de referencia.

\section{Resultados}

La tasa de respuesta es de un 100\%. Al mismo tiempo, el porcentaje de aquellos participantes que afirman querer introducir el género en su futura docencia es muy alto (un 98'5\%). Pese a ello, creemos necesario formular algunas reservas ya que un buen número de los encuestados (33'3\%) ha sido incapaz de materializar esta supuesta intención en una propuesta concreta. Así, el número de respuestas en blanco o con formulaciones generales o ambiguas es muy alto (23 de un total de 69 encuestas). Es este un tipo de réplica que es más frecuentes entre los alumnos sin formación en género, como muestran los datos recogidos en la tabla 1: casi siete de cada diez estudiantes sin formación es incapaz de responder a esta pregunta, mientras que el número desciende a uno de cada cuatro en el caso de aquellos que sí poseen una formación inicial en género (Tabla 1).

\section{Tabla 1}

Respuestas erróneas en relación a la formación en género recibida.

Fuente: elaboración propia

Estudiantado sin formación

Estudiantado con formación

\begin{tabular}{l|c|c|c|c|}
\hline & Respuestas erróneas & \% dentro del subgrupo & Respuestas erróneas & \% dentro del subgrupo \\
\hline $2016-17$ & 5 & $55^{\prime} 5 \%$ & 6 & $29^{\prime} 7 \%$ \\
\hline $2017-18$ & 7 & $85 \%$ & 5 & $18 \%$ \\
\hline Total & 12 & $68^{\prime} 7 \%$ & 11 & $22^{\prime} 6 \%$ \\
\hline
\end{tabular}


En el estudio de los resultados hemos contemplado dos niveles de análisis:

1) La disciplina de referencia, la época histórica y el ámbito de aplicación de las propuestas diseñadas (político, social, cultural o económico).

2) La relación de las propuestas con las diferentes posibilidades de incorporación de las mujeres al currículo que plantea la bibliografía especializada y que operan en la investigación como categorías.

\section{Ámbito disciplinar y cronológico de las propuestas}

Del total de respuestas analizadas, cuarenta y seis pueden adscribirse a una o a varias de las disciplinas que integran las Ciencias Sociales, con una marcada prevalencia de la Historia y la Historia del Arte (Tabla 2). El factor determinante en este caso parece ser la formación disciplinar previa, ya que tiende a haber una correlación entre los resultados porcentuales de las disciplinas a la que se adscriben las propuestas y los datos de la muestra referentes a la formación disciplinar previa (Tabla 3). Un segundo factor que, a nuestro juicio, privilegia las propuestas de Historia del Arte es el hecho de que el cuestionario ha sido implementado en una asignatura consagrada a la didáctica de la Historia del arte.

\section{Tabla 2}

Disciplina a la que pertenecen las propuestas para incorporar el género. Fuente: elaboración propia

Disciplina a la que pertenecen las propuestas

\begin{tabular}{r|c|c|}
\hline & No & $\%$ \\
\cline { 2 - 3 } Historia & 24 & $52 \%$ \\
\cline { 2 - 3 } Historia del Arte & 18 & $39 \%$ \\
\cline { 2 - 3 } Geografía & 2 & $4,3 \%$ \\
\cline { 2 - 3 } Ciencias Sociales & 2 & $4,3 \%$ \\
\cline { 3 - 3 } Total & 46 & 100 \\
\cline { 2 - 3 } & &
\end{tabular}

\section{Tabla 3}

Comparativa entre el ámbito disciplinar al que se adscriben las propuestas realizadas y el grado de origen del alumnado de la muestra en tanto por cien.

Fuente: elaboración propia

\begin{tabular}{lccc} 
& Historia & $\begin{array}{c}\text { Historia del } \\
\text { Arte }\end{array}$ & Geografía \\
& & $39 \%$ & $5^{\prime} 54 \%$ \\
\hline Disciplina de la propuesta & $52 \%$ & $39 \%$ & $10 \%$ \\
\hline Grado de origen del alumnado & $59^{\prime} 98 \%$ & $21^{\prime} 42 \%$ & \\
\hline
\end{tabular}


Un porcentaje muy elevado de aquellas relativas a la Historia del Arte presentan a la mujer como creadora (44'4\%). A ello, los participantes añaden $\left(16^{\prime} 6 \%\right)$ la necesidad de incorporar al aula las obras de las mujeres artistas (Pollock, 1998; Mayayo, 2003; Hustvedt, 2017). Con un peso relativo similar se incorporan diseños acerca del tratamiento de la figura femenina en las representaciones artísticas $\left(16^{\prime} 6 \%\right)$. Por último, no dejan de ser relevantes pese a su carácter excepcional las alusiones al papel de las mujeres como mecenas del arte o como "musas", los análisis del currículo y de los materiales curriculares o el estudio comparado de dos artistas, masculino y femenino, en un estilo artístico determinado (Tabla 4). En cualquier caso, tan sólo dos propuestas son lo suficientemente concretas como para proporcionar nombres de mujeres artistas (en concreto, los de Sofonisba Anguissola, Clara Peeters, Luisa Roldán y Artemisa Gentileschi).

\section{Tabla 4}

Contenidos de las propuestas para introducir el género en relación a la Historia del Arte en términos absolutos y relativos. Fuente: elaboración propia

\begin{tabular}{l|l|l} 
& № & Porcentaje \\
\hline Mujeres creadoras & 8 & $44^{\prime} 4 \%$ \\
\hline Obras de mujeres creadoras & 3 & $16^{\prime} 6 \%$ \\
\hline Representaciones femeninas & 3 & $16^{\prime} 6 \%$ \\
\hline Mujer como musa & 1 & $5^{\prime} 5 \%$ \\
\hline Mecenas & 1 & $5^{\prime} 5 \%$ \\
\hline Comparativa autoría femenina / masculina & 1 & $5^{\prime} 5 \%$ \\
\hline Ausencia del currículo & 1 & $5^{\prime} 5 \%$ \\
\hline
\end{tabular}

El grado de concreción tiende a ser mayor en el caso de los diseños de Historia o en las escasísimas que pueden ser adscritas a la Geografía. En este último caso, lo que resulta preocupante es que la incorporación del género se produzca tan sólo en relación con la mortalidad femenina y con la mortalidad femenina en relación a la violencia de género, como si el género no implicara a la disciplina en su conjunto (Del Valle, 1991; Bondi, 1993; Wright, 2008 y 2009).

Por lo que respecta al marco cronológico, dos tercios de los diseños incluyen alguna referencia temporal, siendo mayoritarias las que afectan a toda la Historia del Arte o la Edad Contemporánea (25'7\% en ambos casos), pero también a toda la Historia o a la época actual (16'1\% respectivamente). De forma secundaria, hay diseños que pueden ser adscritos a la Edad Moderna (9'6\%) o a la Edad Media (6 $45 \%$ ), al tiempo que no consta ninguna para Prehistoria o la Edad Antigua. Por ámbitos, la prevalencia corresponde a la historia política (39³\%), con una presencia también relevante de las propuestas relacionadas con la sociedad y la vida cotidiana (25\%). 


\section{Las propuestas en relación a las posibilidades de incorporar a las mujeres al currículo de Ciencias Sociales que señala la bibliografía}

Documentamos un total de veintitrés codificaciones que hemos adscrito a una de las cuatro categorías enunciadas en el apartado de metodología: 1) la Historia, la Geografía y la Historia del Arte sin mujeres; 2) con las mujeres; 3) de las mujeres; 4) desde la perspectiva de las mujeres (vid supra). La tabla 5 recoge la relación entre las categorías y las codificaciones, al tiempo que la tabla 6 y el gráfico 1 reflejan el peso cuantitativo de unas y de otras.

\section{Tabla 5}

Codificaciones de las propuestas para incorporar a las mujeres al currículo de ciencias sociales en relación a las categorías.

Fuente: elaboración propia

\begin{tabular}{|c|c|}
\hline $\begin{array}{l}\text { La Historia, la Geografía, la } \\
\text { Historia del Arte sin mujeres }\end{array}$ & $\begin{array}{l}\text { A1. Mujeres invisibilizadas, olvidadas, marginadas } \\
\text { A2. Ausencia de las mujeres del currículo } \\
\text { A3. Androcentrismo (relato, hombres importantes) } \\
\text { A4. Equidad, igualdad, respeto, discriminación } \\
\text { A5. Patriarcalismo }\end{array}$ \\
\hline $\begin{array}{l}\text { La Historia, la Geografía, la } \\
\text { Historia del Arte y las mujeres }\end{array}$ & $\begin{array}{l}\text { B1. Mujer como creadora } \\
\text { B2. Consideración social diferencial de las mujeres } \\
\text { B3. Mujeres importantes } \\
\text { B4. El género como adición a un relato androcéntrico }\end{array}$ \\
\hline $\begin{array}{l}\text { La Historia, la Geografía, la } \\
\text { Historia del Arte de las } \\
\text { mujeres }\end{array}$ & $\begin{array}{l}\text { C1. Los discursos sobre las mujeres. } \\
\text { C2. Las representaciones femeninas } \\
\text { C3. Las mujeres en la sociedad y la vida cotidiana } \\
\text { C4. La participación femenina en las revoluciones } \\
\text { C5. Hitos en la historia de las mujeres } \\
\text { C6. El género como elemento transversal }\end{array}$ \\
\hline $\begin{array}{l}\text { Las Ciencias Sociales desde la } \\
\text { perspectiva de las mujeres }\end{array}$ & $\begin{array}{l}\text { D1. El género en relación a la historiografía } \\
\text { D2. Género como perspectiva o enfoque } \\
\text { D3. Identidades } \\
\text { D4. Identidades no binarias- Queer, LGTBI+, cis... } \\
\text { D5. Masculinidades } \\
\text { D6. Las mujeres como parte del discurso habitual de las } \\
\text { Ciencias Sociales } \\
\text { D7. Textos y obras artísticas de autoría femenina } \\
\text { D8. Didáctica crítica }\end{array}$ \\
\hline
\end{tabular}




\section{Tabla 6}

Peso relativo de las diferentes categorías en las propuestas para incorporar a las mujeres al currículo de Ciencias Sociales.

Fuente: Elaboración propia

\begin{tabular}{r|c|c|c|c|}
\multicolumn{2}{r}{ Sin mujeres } & Con mujeres & De las mujeres & \multicolumn{2}{c}{$\begin{array}{c}\text { Desde la perspectiva de las } \\
\text { mujeres }\end{array}$} \\
\hline $\begin{array}{r}\text { No de } \\
\text { codificaciones }\end{array}$ & 24 & 24 & 32 & 35 \\
\cline { 5 - 6 }$\%$ & $20^{\prime} 8 \%$ & $20^{\prime} 8 \%$ & $27^{\prime} 8 \%$ & $30^{\prime} 4 \%$ \\
\cline { 5 - 6 }
\end{tabular}

\section{Gráfico 1}

Recurrencia de las distintas codificaciones en relación a las categorías de análisis. Fuente: elaboración propia

\section{Recurrencia de las codificaciones en relación a las categorías de análisis}

10

10

10

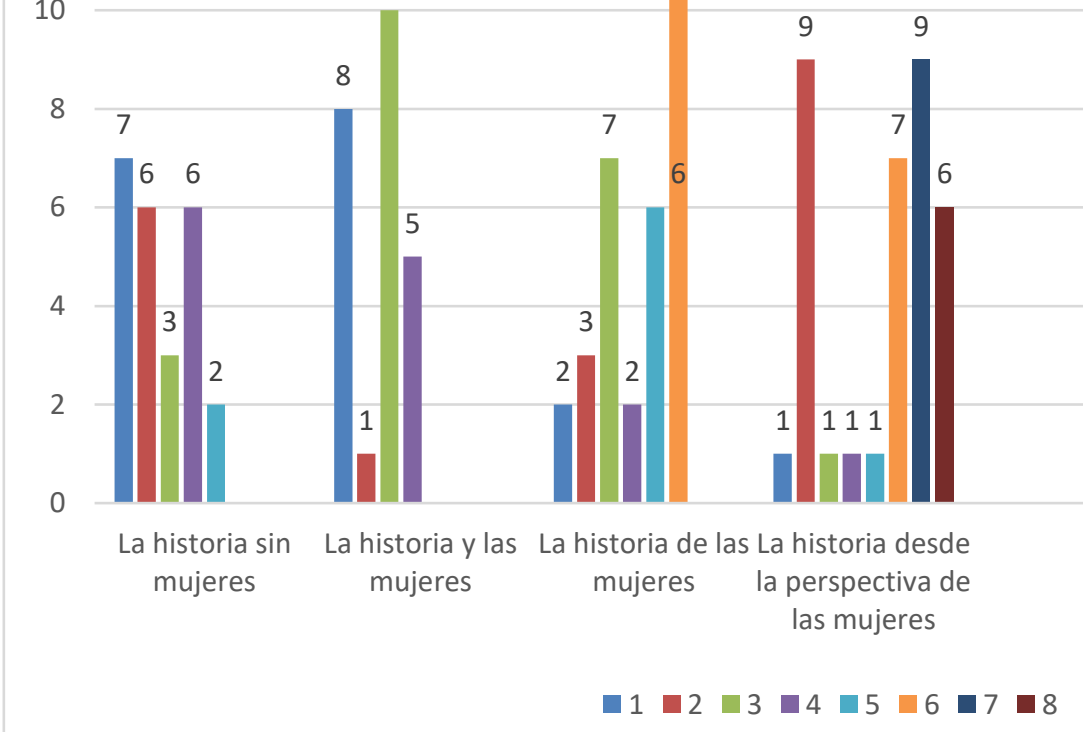

Como puede observarse, entre las codificaciones más utilizadas destacan aquella que reivindica la transversalidad del género (codificación C6, recurrencia de 12), 
pero también la que alude a la presencia de mujeres importantes (codificación B3, recurrencia de 10), el género como perspectiva, punto de vista o enfoque (D2, recurrencia de 9) o la necesidad de incorporar textos literarios y obras de autoría femenina (codificación D7, recurrencia de 9). Igualmente, la importancia de reivindicar la autoría femenina dentro de la Historia del Arte (B1, recurrencia de 8), la necesidad de que las mujeres sean parte del discurso habitual de las Ciencias Sociales (codificación D6, recurrencia de 7) o su presencia en las sociedades del pasado o en la vida cotidiana (C3, recurrencia de 7). Por último, las denuncias de su invisibilidad (A1, recurrencia de 7) o el androcentrismo, el peso del patriarcalismo o aquellas cuestiones relacionadas con una didáctica crítica (codificaciones A3, A5 y D8, todas ellas con una recurrencia de 6).

Por lo que respecta a las categorías, las propuestas de los participantes tienden a situarse más en relación a las categorías $\mathrm{C}$ y $\mathrm{D}$, ya que la suma de ambas vendría a agrupar el 58'6 \% de las respuestas. Las otras dos posibilidades de incorporación de las mujeres al currículo, categorías A y $\mathrm{B}$, aparecen también con una presencia similar (algo por debajo del 21\%), lo que vendría a darnos casi una relación de 40 a 60 entre el primer y el segundo par de categorías.

\section{Las Ciencias Sociales sin las mujeres. La incorporación de las mujeres como una adición al currículo.}

Empezamos con una denuncia: una de cada cinco aportaciones subraya la ausencia de las mujeres del relato de la Historia, la Geografía o la Historia del Arte (categoría A). Contabilizamos aquí las respuestas que subrayan la ausencia o el lugar secundario de las mujeres en el currículo o en los materiales curriculares (codificación A2, recurrencia de 6), su invisibilidad (A1, recurrencia de 7), su androcentrismo (A3, recurrencia de 3) o la prevalencia en ellos de los enfoques patriarcales (A4, recurrencia de 2), además de relacionarlo con la búsqueda de la igualdad o la equidad (A5, recurrencia de 6). Con una presencia relativa similar contabilizamos las propuestas que consideran la incorporación de las mujeres al currículo como adición a unas Ciencias Sociales que siguen definiendo sus contenidos disciplinares en términos masculinos. En este apartado (categoría B), el mayor peso relativo corresponde a las que podríamos denominar "grandes mujeres", que están presentes en una de cada seis respuestas del cuestionario (15'6\% del total de codificaciones). Se trata, en concreto, de las mujeres artistas (codificación B1, recurrencia de 8) o de las "mujeres importantes para la historia" (codificación B3, recurrencia de 10). Son postulados que subrayan que las mujeres sí han tenido relevancia dentro de las Ciencias Sociales, "documentándolas en los mismos espacios que los hombres, especialmente en la cumbre del poder reinas - o de la cultura -escritoras, artistas-" (Bolufer, 2018, p. 50) o de la creación artística (Nochlin 1971, 1989; Parker y Pollock, 1981; Mayayo, 2003). Desde el punto de vista material, los diseños adoptan la forma de adición al 
currículo (codificación B4, recurrencia de 5), planteándose a partir de posibilidades diferentes: una unidad didáctica, un capítulo, un anexo, un taller, una actividad, el estudio de una biografía... Aparece, por otro lado, una única alusión a la valoración social diferencial de hombres y mujeres (codificación B2).

\section{La Historia, la Geografía, la Historia del Arte "de" las mujeres}

Esta tercera categoría (categoría C) incluye las contribuciones de las mujeres en una Historia, Geografía o Historia del Arte todavía androcéntricas, pero que consideran la incorporación de algunos hechos claves relacionados con las mujeres (Woyshiner, 2002). Son planteamientos que destacan, por ejemplo, los grandes hitos de la historia de las mujeres, con especial atención a la lucha de las mismas por conseguir el acceso al poder, en especial en relación con el ejercicio del derecho al voto. En las propuestas de los participantes, aparece en las alusiones al movimiento sufragista, citado hasta en tres ocasiones, pero también a partir de hechos históricos concretos relacionados con la participación de las mujeres estadounidenses en la Segunda Guerra Mundial, el peso de la represión franquista sobre ellas o su presencia en el maquis (codificación C5, recurrencia de 6). También en diseños que aluden al protagonismo de determinados colectivos como las maestras de la segunda república o a la participación femenina en motines, revueltas y revoluciones, siendo citadas de manera específica la revolución francesa, la revolución industrial, la revolución rusa y la revolución liberal en España (C4, recurrencia de 2).

En este nivel de análisis goza de un peso específico considerable el papel de las mujeres en la sociedad y, residualmente, la cotidianidad, lo que ubica a las mujeres en "esferas propias", revalorizando espacios específicos como la familia o la vida cotidiana (codificación D3, frecuencia de 7). Se añaden otros enfoques, ya tradicionales también dentro del género, como son el análisis de los discursos construidos sobre las mujeres a lo largo de la historia (D1, recurrencia de dos) o el tratamiento de la imagen femenina en la historia del arte (D2, recurrencia de 3), a partir de representaciones que "las han pensado, definido, adulado y censurado, regulando sus comportamientos y tratando de fijar su naturaleza" (Bolufer, 2018: 64-65). Sin embargo, la codificación que posee mayor importancia es aquella que niega que la incorporación del género a las ciencias sociales deba hacerse en forma de una adición (C3, recurrencia de 12), que se expresa en términos como los que transcribimos a continuación:

- "En cada unidad didáctica dedicaría un día a ver el temario desde una perspectiva de género, para que puedan comparar y tener una visión más amplia".

- "Lo haría de forma transversal, mostrando los hitos en la historia de las mujeres, a las sufragistas o a personajes relevantes".

- "En cada tema, incluiría un apartado dedicado a las mujeres, por ejemplo, la mujer durante la revolución liberal española".

- "Intentaría incorporarlo a cada uno de los temas". 


\section{Las Ciencias sociales desde la perspectiva de las mujeres}

Contemplamos en este apartado aquellas propuestas que van en la línea de realizar una reescritura de las Ciencias Sociales desde la perspectiva de género. Incluyen elementos como el trabajo directo sobre fuentes o el estudio de creaciones artísticas femeninas. También la valoración de las mujeres en relación a otros colectivos marginalizados, su vinculación con las identidades, la incorporación del género a partir de problemas socialmente relevantes, el uso de un lenguaje inclusivo, las alusiones al colectivo LGTBi+, el empoderamiento femenino o la contraposición, frente a los valores masculinos, de otros, estos femeninos.

La alusión a las identidades, la masculinidad o la concepción del género como construcción cultural e histórica se documentan en tan sólo una ocasión (codificaciones D1, D3, D4 y D5). Poseen, en cambio, un peso mayor las respuestas relacionadas con una didáctica crítica (codificación D5, frecuencia de 6), que plantean el análisis del currículo o los materiales curriculares desde la perspectiva de género, la conexión con el entorno inmediato, la educación en valores, el lenguaje inclusivo o el tratamiento a partir de problemas socialmente relevantes, en concreto la violencia de género. Además, la demanda explícita de crear una asignatura específica de género en el Máster de Profesor/Profesora de Enseñanza Secundaria. A ellas se añaden las voces que abogan por "enfocar el estudio de la historia desde la visión de las mujeres", "incluir el punto de vista femenino" o tratar un mismo hecho histórico o artístico a partir de la comparación de varias fuentes, escritas o artísticas, de autoría femenina y masculina (codificación D7, recurrencia de 9). En una línea similar, algunos participantes abogan por incorporar a las mujeres como parte del discurso habitual de las Ciencias Sociales (codificación D6, recurrencia de 7). Lo hacen en términos como los siguientes: "sin añadirlas como anexo al final de los temas", "no como anexos al margen", "introduciendo de manera equitativa a la mujer en el discurso", "no como apartado separado, sino incluyéndolo en el temario", "de forma integrada en el resto de las explicaciones" ... En su apoyo recurren al soporte material que proporcionan las fuentes de la historia de las mujeres o las obras de arte de autoría femenina, mencionando de forma específica diarios, películas, obras de arte o fuentes literarias (codificación D2, recurrencia de 9).

\section{Conclusión}

Parece innegable que la formación en género de los futuros profesores de secundaria resulta determinante a la hora de poder plantear una propuesta para introducir el género en las clases de Historia, Geografía e Historia del Arte: el 77²\% de aquellos que han recibido formación son capaces de diseñar una intervención, mientras que este porcentaje se reduce hasta el 31'3\% cuando no la han recibido. Por otra parte, confirmamos cómo la formación disciplinar previa condiciona también el diseño de sus propuestas, ya que la mayoría de ellos opta por circunscribir este a su marco disciplinar de referencia, sea Historia, Geografía o Historia del Arte. Por 
último, constatamos la inespecificidad de los diseños que hacen constar, ya que sólo 15 de las 46 formulaciones atribuibles a una determinada disciplina poseen un grado de concreción suficiente que las haga referirse a un caso o ejemplo concreto $\left(21^{\prime} 7 \%\right.$ del total). Cuando intentan responder, los participantes apuestan de forma genérica por la transversalidad del género o por la necesidad de incorporar a las mujeres dentro del discurso habitual de las Ciencias Sociales, aunque sin descartar totalmente la mera adición al currículo. Para ello, señalan como recursos, de manera inconcreta, textos literarios y obras artísticas de autoría femenina, mientras que, en relación con el sujeto y la agencia dentro de la Historia, la Historia del Arte y la Geografía, siguen destacando a los personajes relevantes, esta vez femeninos.

Todo ello nos hace pensar que aún queda mucho por hacer en materia de género y formación del profesorado. Como señalábamos en la introducción, el nuestro sigue siendo un sistema educativo sexista que perpetúa el androcentrismo a través de distintos mecanismos. Uno de ellos es la exclusión de las aportaciones de las mujeres y del tratamiento del género en los currículos. Otro, la ficción de que la igualdad entre hombres y mujeres es ya algo conseguido. Se añade, por último y de forma no banal, la ausencia de formación específica en género de muchos de los futuros docentes de educación primaria o secundaria, lo que los convierte en perfectos perpetuadores de un sistema de dominación y discriminación en el que ellos mismos se hallan inmersos.

\section{Referencias}

Alaminos, A. y Castejón, J. L. (2006). Elaboración, análisis e interpretación de encuestas, cuestionarios y escalas de opinión. Universidad de Alicante.

Álvarez-Gayou, J. L. (2003). Cómo hacer investigación cualitativa. Fundamentos y metodología. Paidós.

Anguita, R. (2011). El reto de la formación del profesorado para la igualdad. REIFOP, 14 (1), 43-51.

Anguita, R. \& Torrego, L. (2009). Género, educación y formación del profesorado. Retos y soluciones, Revista interuniversitaria de formación del profesorado, 64, 17-26.

Berger, J. (1972). Ways of Seeing. Londres, Penguin [trad. esp.: Modos de ver, Gustavo Gili, 2001].

Bolufer, M. (2018). Mujeres y hombres en la historia. Una propuesta historiográfica y docente. Comares.

Bondi, L. (1993). Gender and geography: crossing boundaries. Progress in Human Geography, 17

(2),

241-246. https://doi.org/10.1177/030913259301700208

Cohen, L., Manion, L. y Morrison, K. (2007). Research methods in education. Routledge.

Creswell, J. W. (2014). Research design. Qualitative, quantitative and mixed methods Approaches. Sage. 
Crocco, M. S. (1997). Making time for women's history. When your survey course is already filled to overflowing. Social Education, 61 (1), 32-37.

Crocco, M. S. (2006). Gender and social education: What's the problem? En Ross, E.W. (Ed.), The social studies curriculum: Purposes, problems, and possibilities (pp. 171-193). State University of New York Press.

Del Valle, T. (1991). El espacio y el tiempo en las relaciones de género. KOBIE. Serie Antropología Cultural, V, 223-236.

Donoso, T. \& Velázquez, A. (2013) ¿Por qué una propuesta de formación en perspectiva de género en el ámbito universitario? Profesorado. Revista de currículum y formación del profesorado, 17 (1), 71-88.

Fernández, A. (2001). Las mujeres en la enseñanza de las Ciencias Sociales. Síntesis.

Ibeas, N. \& Garrido, B. (2018). La perspectiva de género en la educación aragonesa. Prensas de la Universidad de Zaragoza.

Giroux, H. A. (1990). Los profesores como intelectuales. Hacia una pedagogía crítica del aprendizaje. Paidós/MEC.

González-Sanmamed, M. (2015). El practicum en la formación del profesorado de secundaria. Revista española de pedagogía, 261, 301-319.

Hernández, Maㅡ. \&. \& Carrasco, V. (2012). Percepciones de los estudiantes del Máster en Formación del Profesorado de Educación Secundaria: fortalezas y debilidades del nuevo modelo formativo. Enseñanza \& Teaching (30), 2, 127 152.

Jaramillo, C. (1999). Formación del profesorado: igualdad de oportunidades entre chicas y chicos. Madrid: Ministerio de Trabajo y Asuntos Sociales. Instituto de la mujer, 1999.

Jackson, P. W. (1999). La vida en las aulas. Morata.

Hustvedt, S. (2017). La mujer que mira a los hombres que miran a las mujeres. Ensayos sobre feminismo arte y ciencia. Seix Barral.

Kimmel, M. S. (1997). Masculinity as Homophobia: Fear, Shame and Silence in the Construction of Gender Identity. En M. M. Gergen \& S. N. Davis (Eds.), Toward a new psychology of gender (pp. 223-242). Taylor \& Francis/Routledge.

Lerner, G. (1979). The majority finds its past: placing women in history. Oxford University Press.

Lerner, G. (1993). The creation of feminist consciousness. Oxford University Press.

López-F. Cao, M. (2001). Modelos, conductas y estereotipos femeninos en la creación icónica. En Fernández, A., Las mujeres en la enseñanza de las CCSS (pp. 169205). Síntesis.

McIntosh, P. (1983). Interactive phases of curricular re-vision. Working Paper, 124.

Manso, J. \& Martín, E. (2014). Valoración del Máster de Formación de Profesorado de Educación Secundaria: estudio de casos en dos universidades. Revista de Educación, 364, 145-169. DOI: 10.4438/1988-592X-RE-2014-364-258

Mayayo, P. (2003). Historias de mujeres, historias del arte. Cátedra.

McIntosh, P. (1983). Interactive phases of curricular re-vision, Working Paper, 124.

Minnich, E. K. (1990). Transforming Knowledge. Temple University Press. 
Moreno,E. (2000). La transmisión de modelos sexistas en la escuela. En SantosGuerra. El harén pedagógico. Curriculum y construcción del género en la escuela (pp. 11-23). Graó.

Mulvey, L. (1989). Fears, Fantasies and the Male Unconscious or 'You Don't Know What is Happening, Do You, Mr Jones?' En L. Mulvey, Visual and other pleasures. Collected writing (pp. 6-13). Macmillan.

Nochlin, L. (1971). Why Have There Been No Great Women Artists? Art News 69, 2239.

Nochlin, L. (1989). Women, Art, and Power and other Essays. Taylor \& Francis.

Nead, L. (1998). El desnudo femenino. Arte, obscenidad y sexualidad. Tecnos.

Pagés, J. y Sant, E. (2012). Las mujeres en la enseñanza de la historia: ¿Hasta cuándo serán invisibles? Cadernos de Pesquisa do Cdhis, 25 (1), 91-117. http://www.seer.ufu.br/index.php/cdhis/article/view/20957

Parker y Pollock (1981). Old Mistresses. Routledge \& Kegan Paul PLC.

Pollock, G. (1988). Vision and Difference. Femininity, Feminism and Histories of Art. Routledge Classics.

Rausell, H. \& Talavera, M. (2017). Dificultades de la coeducación en la formación del profesorado". Feminismos, 29, 329-345. DOI 10.14198/fem.2017.29.13

Rebollar Sánchez, E. (2013). El género en los planes de estudio de los grados de educación de las universidades públicas españolas. Universidad Autónoma de Barcelona (Tesis doctoral).

Ross, E. W. (ed.) (1994). Reflective practice in social studies education. National Council for the Social Studies, 88, 5-10.

Simons, H. (2011). El estudio de caso: Teoría y práctica. Morata.

Stake, R. E. (2007). Investigación con estudio de casos. Morata.

Woyshner, C. (2002). Political history as women's history: toward a more inclusive curriculum. Theory and Research in Social Education, 30 (3), 354-380. https://doi.org/10.1080/00933104.2002.10473201

Taylor, S. J. y Bodgan, R. (1984). Introducción a los métodos cualitativos de investigación. La búsqueda de significados. Paidós.

Wright, M. W. (2008). Gender and geography: knowledge and activism across the intimate global. Progress in Human Geography, 33 (3), 379-386. https://doi.org/10.1177/0309132508090981

Wright, M. W. (2009). Gender and geography: bridging the gap - feminist, queer and the geographical imaginary. Progress in Human Geography, 34 (1), 56-66. https://doi.org/10.1177/0309132509105008 
